\title{
New mechanism of oral immunity to mucosal candidiasis in hyper-lgE syndrome
}

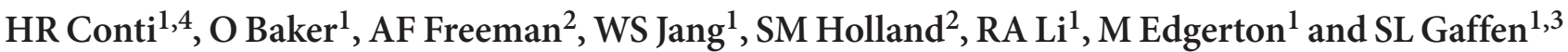

Oropharyngeal candidiasis (OPC, thrush) is an opportunistic infection caused by the commensal fungus Candida albicans. An understanding of immunity to Candida has recently begun to unfold with the identification of fungal patternrecognition receptors such as C-type lectin receptors, which trigger protective T-helper (Th)17 responses in the mucosa. Hyper-IgE syndrome (HIES/Job's syndrome) is a rare congenital immunodeficiency characterized by dominant-negative mutations in signal transducer and activator of transcription 3, which is downstream of the Th17-inductive cytokines interleukin (IL)-6 and IL-23, and hence patients with HIES exhibit dramatic Th17 deficits. HIES patients develop oral and mucocutaneous candidiasis, supporting a protective role for Th17 cells in immunity to OPC. However, the Th17dependent mechanisms of antifungal immunity in OPC are still poorly defined. An often unappreciated aspect of oral immunity is saliva, which is rich in antimicrobial proteins (AMPs) and exerts direct antifungal activity. In this study, we show that HIES patients show significant impairment in salivary AMPs, including $\beta$-defensin 2 and Histatins. This tightly correlates with reduced candidacidal activity of saliva and concomitantly elevated colonization with Candida. Moreover, IL-17 induces histatins in cultured salivary gland cells. This is the first demonstration that HIES is associated with defective salivary activity, and provides a mechanism for the severe susceptibility of these patients to OPC.

\section{INTRODUCTION}

Oropharyngeal candidiasis (OPC, thrush) is a frequent opportunistic infection associated with defects in cell-mediated immunity, particularly in AIDS (acquired immunodeficiency syndrome) and following chemotherapy. OPC is most frequently caused by the commensal yeast Candida albicans. The same organism is responsible for vaginal candidiasis and a disseminated form of blood-borne candidemia. Disseminated candidiasis is the fourth most common hospital-acquired infection, and is associated with severe mortality (averaging $~ 40 \%) .{ }^{1}$ However, patients with AIDS are not highly prone to either vaginal or disseminated candidiasis, ${ }^{2,3}$ and therefore immunity to OPC seems to vary by anatomical site.

The high prevalence of OPC in HIV (human immunodeficiency virus)/AIDS strongly implicates CD4 + T-helper (Th) cells in protecting against this disease. There are three main types of Th cells, namely Th1 (interferon- $\gamma$ secreting), Th2 (interleukin (IL)-4, IL-5, and IL-13 secreting), and Th17 (IL-17 and IL-22 secreting). ${ }^{4}$ Although immunity to OPC was long considered to be dominantly mediated by Th1 cells, recent evidence strongly indicates that this is instead the province of Th17 cells. ${ }^{5-7}$ In particular, Th17-deficient mice but not Th1-deficient mice are susceptible to OPC. ${ }^{5}$ Similarly, mice and humans deficient in pattern-recognition receptors that stimulate IL-23-dependent Th17 responses such as dectins- 1 and -2 , the nod-like receptor, pyrin domain-containing 3 inflammasome, or caspase recruitment domain family, member 9 are also susceptible. ${ }^{6,8-12}$ In humans, the majority of Candida-specific memory T cells express IL-17 and the Th17 marker CCR6, ${ }^{13}$ and individuals with neutralizing antibodies to Th17 cytokines due to thymoma or Aire deficiency are highly susceptible to thrush. ${ }^{12,14-16}$ Thus, there is mounting evidence that suggests that IL-17 signaling is essential for immunity to oral candidiasis. However, the specific mechanisms by which IL-17 mediates these effects, particularly in the context of the oral mucosa, are not well defined.

Hyper-IgE/Job's syndrome (HIES) is a rare multisystem immunodeficiency disorder characterized by elevated IgE, eczema, recurrent infections, bone and connective tissue

${ }^{1}$ Department of Oral Biology, University at Buffalo, SUNY, Buffalo, New York, USA. ${ }^{2}$ Laboratory of Clinical Infectious Disease, National Institute of Allergy and Infectious Diseases, National Institutes of Health, Bethesda, Maryland, USA. ${ }^{3}$ Division of Rheumatology and Clinical Immunology, Department of Medicine, University of Pittsburgh,

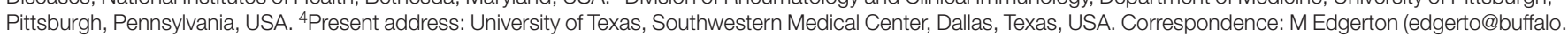
edu) or SL Gaffen (sig65@pitt.edu) 
abnormalities, and lesions of the palate and dorsal tongue. ${ }^{17}$ Patients with HIES exhibit a high prevalence of mucosal and skin infections, particularly "cold" staphylococcal abscesses and mucocutaneous and oral candidiasis. ${ }^{18}$ However, these patients only rarely experience disseminated microbial infections, ${ }^{19}$ indicating that the distinct arms of immunity operate in mucosal vs. systemic forms of the disease. The major genetic defects in autosomal-dominant HIES were recently shown to be dominant-negative mutations in the signal transducer and activator of transcription 3 (STAT3) transcription factor. ${ }^{18,20}$ STAT3 is a downstream effector of Th17-inductive cytokines, including IL-6, IL-21, and IL-23, and is essential for differentiation of Th17 cells. ${ }^{21,22}$ STAT3 is also activated by IL-22, another cytokine produced by Th17 cells. In mice, Th17 cells are essential for protection against extracellular microorganisms, including Staphylococcal species and C. albicans. ${ }^{23,24}$ Th17 cells are enriched at the mucosal surfaces and the skin, and appear to be preferentially lost in HIV/AIDS, ${ }^{25}$ potentially explaining the spectrum of opportunistic infections. The lack of Th17 cells in HIES at least partially explains the susceptibility of these patients to mucosal infections, such as thrush. ${ }^{26}$ However, given the pleiotropic nature of STAT3, there may be additional deficits that contribute to the severe susceptibility of HIES patients to candidiasis.

Saliva is an important component of immunity in the oral cavity. In addition to roles in lubrication, digestion, and remineralization, saliva provides a vital first line of defense against C. albicans. ${ }^{27}$ Saliva is highly enriched in antimicrobial proteins (AMPs), such as $\beta$-defensins and histatins (Hsts), which exert direct candidacidal activity and serve to limit $C$. albicans overgrowth and attachment to the oral epithelium. ${ }^{28}$ Saliva also contains agglutinins that bind microorganisms and allow their clearance by swallowing to reduce microbial load. The importance of saliva as an antifungal immune component is starkly illustrated by the high risk of OPC in settings of impaired salivary gland function, such as patients with Sjögren's syndrome or following head/neck irradiation. ${ }^{1}$ Drugs that cause xerostomia also predispose to OPC. However, to date, there are few connections between salivary function and the Th17 axis of immunity.

We recently reported that mice deficient in Th17 cells due to a knockout of IL-23 or IL-17 receptor components are highly susceptible to OPC. ${ }^{5,29}$ An unexpected observation was that saliva obtained from IL-23 - / - and IL-17 receptor (IL-17RA) - / - mice exhibits a decreased ability to kill C. albicans in vitro. ${ }^{5}$ To determine whether the IL-23/Th17 pathway regulates salivary fungicidal activity in humans, we evaluated the candidacidal capacity of saliva samples obtained from HIES patients. In this study, we show that a STAT3 deficiency in HIES is associated with reduced salivary antifungal activity, correlating with reduced expression of specific antimicrobial effectors. This finding provides a direct and novel mechanism by which susceptibility to OPC may occur in HIES. Moreover, this work implicates the IL-23/STAT3 pathway in regulating the antifungal properties of saliva in humans.

\section{RESULTS}

To determine whether Th17-deficient humans show a reduction in salivary antifungal activity as we had previously reported in
IL-23 - / - mice, ${ }^{5}$ we evaluated the candidacidal activity of saliva samples obtained from HIES patients ranging in age from 12 to 53 years. All had common mutations in STAT3, and two-thirds were taking prophylactic antifungal medications (Table 1). Before processing for the candidacidal assay, $25 \mu \mathrm{l}$ of whole saliva from each patient was plated for C. albicans colony enumeration to assess baseline colonization. In healthy controls, C. albicans colonized the oral cavity at low levels, typical of previous observations. ${ }^{30}$ In contrast, there was a much higher level of oral Candida carriage in the HIES cohort, with 7 of 12 patients having fungal burdens exceeding 1,000 $\mathrm{CFU} \mathrm{ml}^{-1}$ (Figure 1a, Table 1).

To assess antifungal activity, endogenous oral flora was removed by centrifugation (confirmed by re-plating) and incubated with a reference strain of C. albicans. As expected, saliva obtained from controls showed robust candidacidal activity (68.6 $\pm 7.74 \%$; Figure 1b). In contrast, saliva obtained from most HIES patients showed significantly reduced candidacidal properties (38.48 $\pm 11.6 \%)$. This was a reproducible finding, because in a few instances, we were able to analyze replicate samples obtained from the same patients at widely separated intervals ( $>2$ months). Candidacidal activity was remarkably consistent between individuals (Supplementary Figure 1 online). Accordingly, salivary antifungal activity is reduced in HIES patients, which correlates with a high oral carriage of C. albicans.

Many HIES patients in this study were taking prophylactic antifungal medications at the time of sample collection (Table 1). Antifungal drug usage did not seem to correlate with reduced fungal burden, as C. albicans was recovered in similar levels from patients regardless of whether they were taking antifungal medications (Figure 1a and $\mathbf{b}$ ). However, antifungal usage did not seem to residually augment the killing properties of saliva, as there was no significant difference in candidacidal activity in samples acquired from patients taking antifungal drugs (Figure 1c). Although the number of samples is admittedly small due to the rarity of this mutation (estimated to be $<1 \times 10^{6}$ ), this finding suggests that susceptibility to Candida carriage and resistance to OPC is not fully prevented by antifungal medications.

Saliva is extremely rich in AMPs. ${ }^{31}$ Th17 cytokines such as IL-17 and IL-22 regulate various AMPs such as defensins, calprotectins, and cathelicidins in mucosal epithelial cells and keratinocytes. ${ }^{32,33}$ In these studies, addition of protease inhibitors (PIs) to the saliva samples was required to preserve antifungal activity during shipping, suggesting that the antifungal effector is a protein. In the absence of PI, saliva obtained from controls and HIES patients showed low fungicidal activity $(3.62 \pm 1.12 \%$ and $3.83 \pm 3.30 \%$, respectively). Addition of PI increased the killing activity of HIES saliva moderately, but was far less than control levels, indicating that saliva obtained from HIES patients is deficient in AMP activity (Figure 1b). Interestingly, HIES whole saliva (with PI) contained a mean of 3.6-fold higher levels of total protein than did control saliva (Figure 2a). This finding may be suggestive of a defect in salivary secretion, resulting in a higher overall concentration of salivary components. We also noted anecdotally that many HIES patients had trouble producing 
Table 1 Patient characteristics (Figures 1 and 2a-d)

\begin{tabular}{|c|c|c|c|c|}
\hline Patient ID no. & Gender/age (years) & STAT3 mutation & Antifungal usage & Fungal carriage \\
\hline HIES-2 & $\mathrm{M} / 18$ & V637L/SH2 & $\mathrm{N}$ & High \\
\hline HIES-4 & $\mathrm{F} / 12$ & V637M/SH2 & Y & High \\
\hline HIES-5 & $\mathrm{M} / 26$ & R382W/DNA binding & Y & None \\
\hline HIES-8 & $M / 53$ & Y657C/SH2 & Y & None \\
\hline HIES-9 & $F / 39$ & V637M/SH2 & Y & High \\
\hline HIES-10 & $\mathrm{F} / 35$ & $\mathrm{~S} 668 \mathrm{~F} / \mathrm{SH} 2$ & Y & High \\
\hline HIES-11 & $F / 34$ & V637M/SH2 & N & Low \\
\hline Control-3 & $\mathrm{F} / 49$ & - & $\mathrm{N}$ & Low \\
\hline Control-4 & $\mathrm{M} / 46$ & - & $\mathrm{N}$ & None \\
\hline Control-5 & $\mathrm{M} / 19$ & - & N & None \\
\hline Control-6 & $M / 16$ & - & $\mathrm{N}$ & None \\
\hline Control-7 & $\mathrm{F} / 10$ & - & $N$ & None \\
\hline Control-8 & $F / 28$ & - & $\mathrm{N}$ & Low \\
\hline Control-9 & Adult & - & $\mathrm{N}$ & None \\
\hline Control-10 & Adult & - & N & Low \\
\hline Control-11 & $\mathrm{F} / 57$ & - & N & None \\
\hline Control-18 & $F / 41$ & - & N & None \\
\hline Control-19 & $F / 38$ & - & N & None \\
\hline Control-20 & $\mathrm{M} / 50$ & - & N & None \\
\hline Control-21 & $\mathrm{F} / 39$ & - & $\mathrm{N}$ & Low \\
\hline
\end{tabular}

Abbreviations: F, female; HIES, hyper-IgE syndrome; M, male; STAT3, signal transducer and activator of transcription 3.

HIES or control subjects used in Figures 1 and $\mathbf{2 a}$ - $\mathbf{d}$ are indicated by gender, age, STAT3 mutation, use of antifungal drugs (yes/no) and baseline fungal carriage (none $=$ no detectable oral carriage; low $=1-200 \mathrm{CFU} \mathrm{ml}^{-1}$; high $>200 \mathrm{CFU} \mathrm{ml}^{-1}$ ). In a few instances in which the age of the subject was unknown, we indicate "adult." It must also be noted that serum creatinine levels were assessed for all patients, and found to be within the normal range (data not shown).

saliva, although no formal studies were carried out to verify this systematically (AF, unpublished observations). Collectively, these observations are strongly suggestive of a salivary gland defect in HIES, which is manifested in a reduced ability to control oral fungal infections.

There are numerous AMPs found in saliva, including defensins, Hst, and secretory leukocyte PI (SLPI). ${ }^{34,35}$ In murine OPC, the impaired expression of $\beta$-defensin (BD) 3 in IL-17RA - / - mice correlates with OPC susceptibility. ${ }^{5}$ Accordingly, we first evaluated the expression of the human analog of BD3, BD2, in HIES or control saliva, by ELISA (enzymelinked immunosorbent assay). Strikingly, levels of BD2 per ml of saliva were decreased 3.87-fold in HIES patients compared with healthy subjects (Figure 2b). Thus, HIES saliva has reduced expression of BD2, consistent with the presence of two predicted STAT3-binding sites in its promoter (S Velichko and R Wu, personal communication).

Histatins are histidine-rich cationic proteins found in human parotid and submandibular secretions, which exhibit potent bactericidal and fungicidal activities. ${ }^{36}$ There are two Hst gene products, namely Hst 1 and Hst 3. Hst 2 arises from autoproteolytic cleavage of Hst 1, whereas Hst 4-Hst 12 are successive degradation products of Hst 3. All Hst isoforms exhibit anticandidal activity. Whereas Hst 5 has the most potent antifungal 

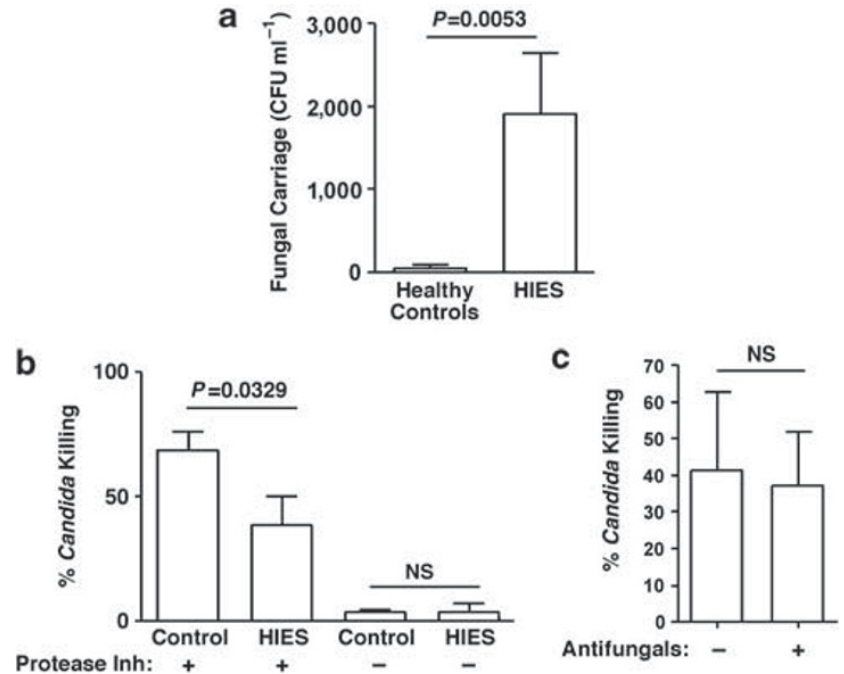

Figure 1 Increased colonization and decreased candidacidal activity in HIES saliva. (a) Fungal carriage in HIES or control saliva. In all, $25 \mu \mathrm{l}$ saliva obtained from healthy controls $(n=21)$ or from HIES patients ( $n=15$, Table 1) was analyzed for endogenous Candida albicans colonization by plating on yeast peptone dextrose in triplicate. \pm s.e.m. $P=0.0053$, two-tailed unpaired $t$-test. (b) Candida killing in HIES or control saliva. Saliva obtained from indicated cohorts was centrifuged to remove endogenous microbes, incubated with $10^{6} \mathrm{C}$. albicans cells (reference strain CAF2-1) for $1 \mathrm{~h}$ at $37^{\circ} \mathrm{C}$ in triplicate, and plated on YPD for colony enumeration as a percentage of a PBS control. A PI cocktail was included at the time of collection where indicated (control, $n=21$; HIES, $n=13$ ). \pm s.e.m. $P=0.0329$, two-tailed unpaired $t$-test. (c) Antifungal medication does not affect the candidacidal activity of saliva. Percentage killing in patients on prophylactic antifungal drugs $(n=9)$ compared with no drugs $(n=4)$. Data are not significant by two-tailed unpaired $t$-test. Data include analysis of all samples from Table 1. HIES, hyperIgE syndrome; NS, not significant; PBS, phosphate-buffered saline; PI, protease inhibitor.

activity, Hst 1 is the most resistant to proteolytic degradation. ${ }^{28}$ There are no available antibodies to Hsts for use in western blots. Therefore, to quantify Hsts, whole saliva was resolved on acidic PAGE, stained, and quantified by image densitometry (Figure $2 \mathbf{c}$ and $\mathbf{d}) .{ }^{37}$ All Hst isoforms were significantly reduced in HIES compared with controls (Figure 2d).

In contrast to BD2 and Hsts, levels of SLPI, another AMP with inhibitory growth effects on C. albicans, were significantly increased (fivefold) in HIES saliva (Figure 2e, Table 2). This may be a compensatory response to the loss of AMP activity, but is consistent with findings in HIV patients in whom a history of oral candidiasis was associated with elevated SLPI levels. ${ }^{34}$ Thus, although some AMPs in saliva such as BD2 and Hsts are indeed negatively impacted by HIES status, some such as SLPI are elevated.

Although most HIES patients exhibited reduced salivary candidacidal activity and concomitantly reduced $\mathrm{BD} 2$ and Hst levels, there were exceptions. In particular, saliva from HIES-8 showed high killing activity on two separate occasions, and had normal levels of Histatin proteins, particularly Hst 1 (Figure 2c, lanes 9 and 10, Supplementary Figure 1 online). This individual was taking antifungal medication (Table $\mathbf{1}$ ) and showed no baseline oral fungal carriage, supporting a role for salivary AMPs in limiting oral Candida colonization/infection. This patient has a typical mutation in the SH2 domain of STAT3 and a history of OPC and onychomycosis. Unlike typical HIES cases, however, he has also experienced several disseminated fungal infections (Histoplasmosis and Cryptococcus) ${ }^{38}$ It is unclear why this patient with a common HIES mutation would have improved candidal killing and normal Histatin levels. ${ }^{38}$ Nonetheless, such outlier patients highlight the fact that immunity to fungi occurs at many levels, ${ }^{16}$ and individual genetic polymorphisms probably exert a significant influence even in the setting of a primary immunodeficiency, such as HIES.

To determine whether Th17 cytokines exert direct effects on salivary secretory cells, we tested the effects of these cytokines on an in vitro system using the human submandibular gland (HSG) cell line. HSG cells are derived from intercalated ductal cells, ${ }^{39}$ which differentiate to give rise to the acinar and myoepithelial cells of the salivary gland. Under normal culture conditions, HSG cells present an undifferentiated epithelial-like morphology. However, when cultured on complex extracellular matrices such as Matrigel, they show morphological differentiation and induce expression of salivary gland differentiation markers, such as vimentin, salivary cystatin, and $\alpha$-amylase. ${ }^{40,41}$ To determine the capacity of salivary cells to respond to Th17 cytokines, HSG cells were grown on growth factor-reduced-Matrigel for 2 days and treated with IL-17, tumor necrosis factor- $\alpha$, or IL-22 for $48 \mathrm{~h}$. The expression of Hsts was then assessed by acidic PAGE. IL-17 treatment reproducibly and significantly enhanced the expression of Hst 1 in these cultures, although this required fairly high doses (ranging from 200 to $500 \mathrm{ng} \mathrm{ml}^{-1}$ depending on the experiment) (Figure 3a and $\mathbf{b}$ ). In some instances, weak expression of Hst 5 could also be seen in IL-17-stimulated samples (data not shown). However, IL-22 and tumor necrosis factor- $\alpha$ did not induce significant Hst 1 expression in HSG cultures, nor did tumor necrosis factor- $\alpha$ or IL-22 synergize with IL-17 to enhance upregulation of Hst 1 (as they do for many other IL-17-regulated genes $^{42-45}$ ). Therefore, IL-17 has the potential to directly impact the expression of AMPs in the human salivary gland tissue.

\section{DISCUSSION}

In this report, we show for the first time that HIES patients exhibit reduced candidacidal activity in saliva. This correlates with reduced expression of certain AMPs with antifungal activity, namely BD2 and Hsts. This work is a direct translational study, inspired by our previous findings that saliva from IL23 - / - (Th17-deficient) mice shows starkly reduced candidacidal activity in vitro. ${ }^{5}$

How does STAT3 deficiency in HIES lead to salivary impairment? One possibility is that activated Th17 cells influence AMP expression in the salivary glands and buccal mucosa. This model is supported by findings that $\mathrm{BD} 3$ (the mouse ortholog of $\mathrm{BD} 2$ ) is dramatically reduced in IL-17RA $-/-$ mice $^{5}$ and that IL-17 directly activates the promoters for mouse $\mathrm{BD} 3$ and human $\mathrm{BD} 2 .{ }^{46}$ In some ways, the salivary impairment in HIES is reminiscent of Sjögren's syndrome, in that patients are susceptible to OPC because of reduced salivary function. Moreover, it has been reported that Act 1 - / - mice (which are defective in IL-17R signaling) have a Sjögren's syndrome-like phenotype. ${ }^{47}$ However, we observed no 

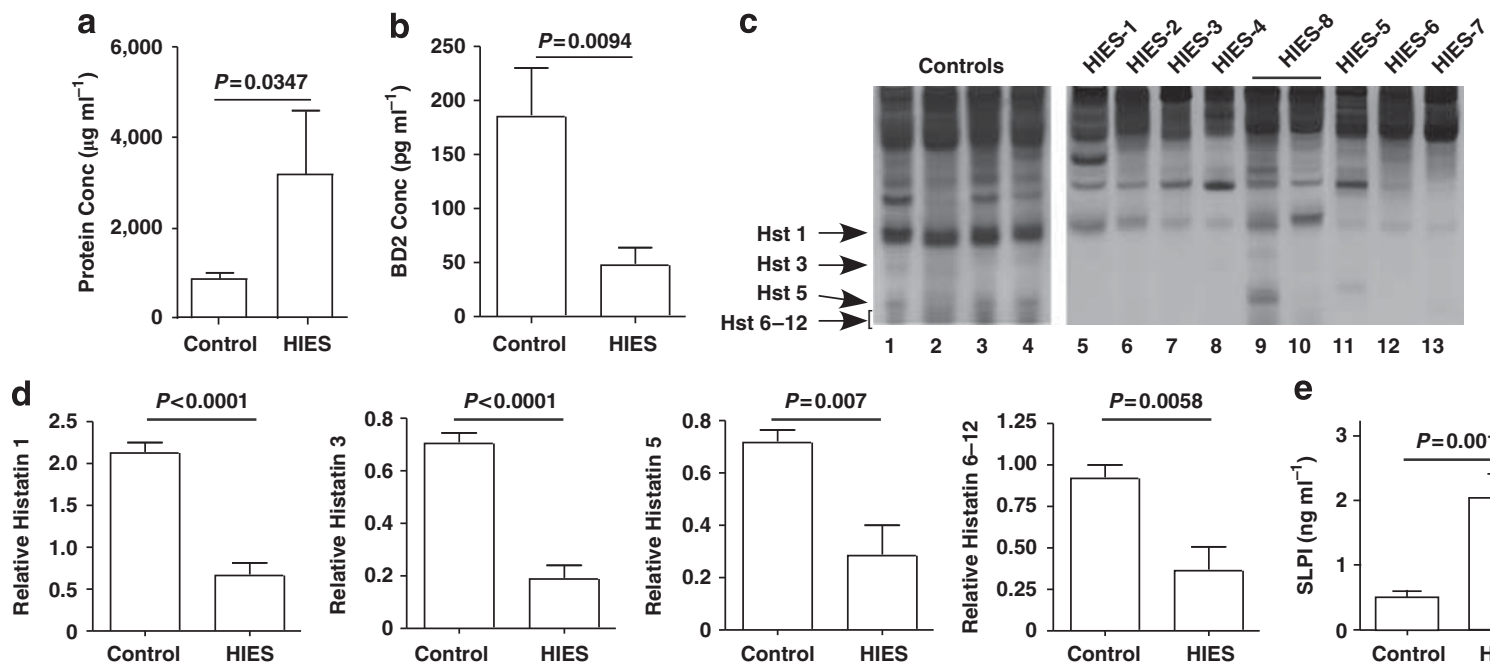

e

Figure 2 Decreased antimicrobial protein expression in HIES saliva. (a) Increased total protein levels in HIES saliva. Total salivary protein concentrations (controls, $n=24$; HIES, $n=14$ ) were assessed by BCA assay in samples treated with PI. Two-tailed unpaired $t$-test. (b) Decreased BD2 levels in HIES saliva. Saliva samples obtained from 18 controls and 12 HIES patients (Table 1) were concentrated on Centricon exclusion columns and analyzed for BD2 by ELISA. $P=0.0094$ by two-tailed unpaired $t$-test. Data were normalized to total protein content. (c) Histatin levels in HIES saliva. Salivary histatin levels in controls $(n=13)$ or HIES subjects $(n=11)$ were analyzed by acid-urea PAGE. Representative samples are shown, with migration of Hst 1, Hst 3, and Hst 5 indicated. (d) Decreased Hst levels in HIES. Densitometric analysis of Histatin bands analyzed as represented in panel c. $P$-values are indicated by two-tailed $t$-test. (e) SLPI levels are increased in HIES. Nine HIES and nine control saliva samples (Table 2) were analyzed for SLPI by ELISA in triplicate. $P$-value by two-tailed $t$-test is indicated. BCA, bicinchoninic acid; BD2, $\beta$-defensin 2; ELISA, enzyme-linked immunosorbent assay; HIES, hyper-IgE syndrome; Hst, histatin; PI, protease inhibitor; SLPI, secretory leukocyte PI.

lymphocytic infiltration or anatomical abnormalities in murine IL-23 - / - salivary glands, despite their reduced candidacidal activity in vitro (HRC, unpublished data). IL-23-/ - mice show reduced candidacidal activity even before exposure to Candida, and therefore the impairment may reflect a developmental effect of IL-23 rather than a Th17-dependent event. Whether this is true in humans or controlled by STAT3 remains unknown.

HIES patients lack Th17 cells, and are strongly prone to developing mucosal candidiasis. ${ }^{17}$ Strikingly, a number of other recent studies in humans have also shown that IL-17 and the Th17 pathway are vital for host defense against mucosal Candida infections (reviewed in ref. 12). For example, humans with defects in the PRR Dectin-1 or its downstream signaling effector caspase recruitment domain family, member 9 are prone to mucosal candidiasis. ${ }^{8,10}$ Signaling through this pathway and through IL-17RA also protects against disseminated candidiasis in mice, although the effects on OPC are not known. ${ }^{11,48-51}$ Similarly, humans with defects in central tolerance due to Aire deficiency autoimmune polyglandular syndrome- 1 or autoimmune polyendocrinopathy-candidiasis-ectodermal develop mucosal candidiasis, which is linked to neutralizing antibodies to Th17 cytokines, including IL-17A, IL-17F, and IL-22. ${ }^{12,14,15}$ In this study, we show that IL-17A but not IL-22 has direct effects on HSG cells (Figure 3), suggesting that a loss of Th17 cells (or, at least, STAT3 signaling) in HIES may compromise the ability of the salivary gland to produce Histatins, and possibly other proteins that contribute to controlling the growth of Candida in the oral cavity.

In this study, not all control subjects showed high levels of salivary $\mathrm{BD} 2$ or Hst expression, and salivary killing of C. albicans varies widely among healthy individuals. ${ }^{30}$ Polymorphisms in
Table 2 Patient characteristics (Figure 2e)

\begin{tabular}{|c|c|c|c|}
\hline Patient ID no. & $\begin{array}{c}\text { Gender/age } \\
\text { (years) }\end{array}$ & $\begin{array}{c}\text { STAT3 } \\
\text { mutation }\end{array}$ & $\begin{array}{c}\text { Antifungal } \\
\text { usage }\end{array}$ \\
\hline HIES-13 & $M / 31$ & $\begin{array}{l}1110(-2) A- \\
G / \Delta E x o n 12\end{array}$ & $N$ \\
\hline HIES-14 & $F / 35$ & S668F/SH2 & $N$ \\
\hline HIES-15 & $F / 26$ & V637M/SH2 & Y \\
\hline HIES-16 & $F / 35$ & V637M/SH2 & $N$ \\
\hline HIES-17 & $\mathrm{M} / 24$ & $\begin{array}{l}\text { R382W/DNA } \\
\text { binding }\end{array}$ & Y \\
\hline HIES-18 & $\mathrm{M} / 15$ & V463del & $\mathrm{N}$ \\
\hline HIES-19 & $\mathrm{F} / 40$ & V637M/SH2 & Y \\
\hline HIES-20 & $M / 11$ & Y657S/SH2 & $\mathrm{N}$ \\
\hline HIES-21 & $M / 16$ & V637M/SH2 & Y \\
\hline Control-22 & $F / 58$ & NA & NA \\
\hline Control-23 & $\mathrm{F} / 29$ & NA & NA \\
\hline Control-24 & $M / 51$ & NA & NA \\
\hline Control-25 & $F / 32$ & NA & NA \\
\hline Control-26 & $\mathrm{F} / 42$ & NA & NA \\
\hline Control-27 & $\mathrm{F} / 27$ & NA & NA \\
\hline Control-28 & $F / 31$ & NA & NA \\
\hline Control-29 & $F / 39$ & NA & NA \\
\hline Control-30 & $\mathrm{F} / 33$ & NA & NA \\
\hline
\end{tabular}

Abbreviations: F, female; HIES, hyper-IgE syndrome; M, male; NA, not applicable; STAT3, signal transducer and activator of transcription 3; WT, wild type. HIES or control subjects used in Figure $\mathbf{2 e}$ are indicated by gender, age, antifungal usage and STAT3 mutation. We confirmed that the candidacidal activity of these HIES samples was reduced compared with WT controls (data not shown). 

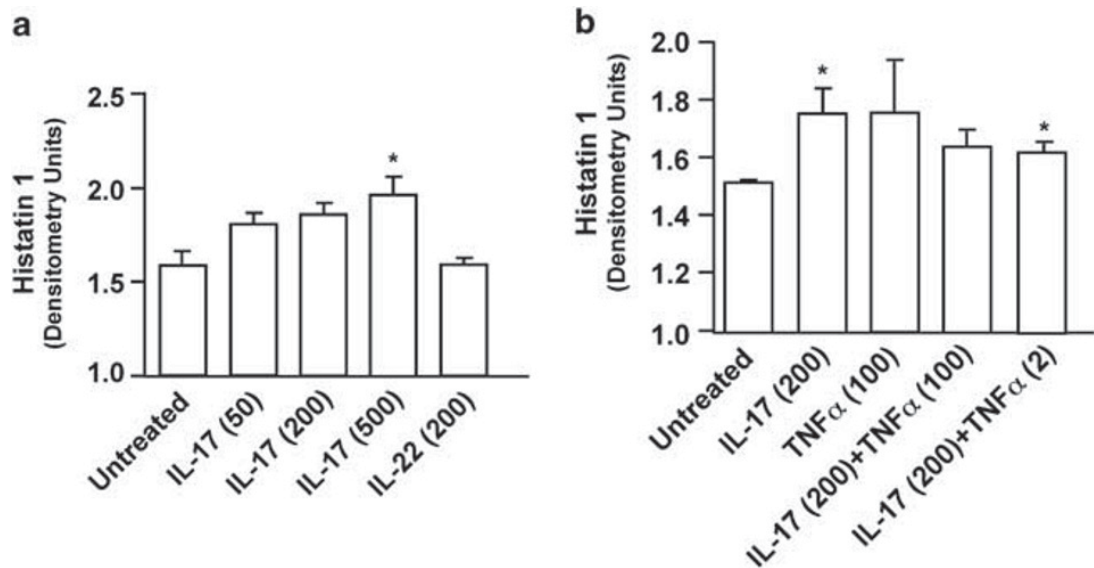

Figure 3 IL-17 induces histatin 1 in HSG cells. HSG cells grown on GFR-Matrigel for 2 days were stimulated for $48 \mathrm{~h}$ with IL-17 (50, 200 , or $\left.500 \mathrm{ng} \mathrm{ml}^{-1}\right)$, TNF $\alpha\left(2\right.$ or $\left.100 \mathrm{ng} \mathrm{ml}^{-1}\right)$, and IL-22 $\left(200 \mathrm{ng} \mathrm{ml}^{-1}\right)$. Lysates were analyzed for histatins by acid-urea SDS-PAGE as described in Figure 2 and quantified by densitometry (units/10). ${ }^{\star} P<0.05$ by two-tailed $t$-test compared with untreated controls. GFR, growth factor reduced; IL, interleukin; Hst, histatin; TNF $\alpha$, tumor necrosis factor- $\alpha$.

genes that regulate AMPs such as NOD2 and IL-23R have been identified in genome wide association study analyses of inflammatory bowel disease and other autoimmune diseases, ${ }^{52}$ and hence it would not be surprising if similar genetic polymorphisms influence immunity in the oral cavity. Indeed, single-nucleotide polymorphisms in human $\beta$-defensin-1 have been linked to oral Candida carriage, ${ }^{53}$ and rare mutations in Dectin- 1 and caspase recruitment domain family, member 9 (which signal in response to Candida antigens) confer susceptibility to mucosal candidiasis. ${ }^{8,10,16,54}$ In addition, there are many common single-nucleotide polymorphisms in loci associated with IL-23R signaling such as STAT3 and IL-12p40, which could account for variability in healthy controls and possibly the outlier HIES patients. ${ }^{55}$

Although many of the patients in the study were taking prophylactic antifungal drugs at the time of the study, the killing capacity of their saliva was reduced compared with controls. The salivary concentrations of the antifungals were not measured in our patient pool. However, it is known that at least for several of the antifungals that patients were receiving (fluconazole, itraconazole, voriconazole), there are measurable salivary concentrations. Conceivably this could have caused increased salivary killing of Candida; however, salivary killing was still diminished in HIES compared with controls, supporting our conclusion that saliva obtained from HIES patients fails to kill C. albicans effectively.

This is, to our knowledge, the first demonstration that HIES is associated with defective salivary activity, and is also the first line of evidence that STAT3 controls AMP expression in the salivary gland and oral mucosa. Although Th17 cells have been implicated in protection against mucosal candidiasis, the specific host defense mechanisms mediated by STAT3 are not well understood. These results provide a specific mechanism by which molecules in this pathway contribute to antifungal host defense at mucosal surfaces.

\section{METHODS}

Candidacidal assays. All saliva samples (HIES and normal controls) were collected by expectoration and shipped in $10 \times$ PI cocktail
(Calbiochem/EMD, cocktail set III, Gibbstown, NJ). For analysis, saliva was centrifuged for $5 \mathrm{~min}$ at $550 \mathrm{~g}$. Saliva was incubated at $37^{\circ} \mathrm{C}$ with $10^{6}$ C. albicans cells (strain CAF2-1) $(1: 1, \mathrm{v} / \mathrm{v})$ for $1 \mathrm{~h}$. Cells were plated in triplicate for colony enumeration. Protocols were approved by the International Review Board at the National Institutes of Health. Both HIES and control subjects signed informed consent.

Statistics. Data were analyzed by Student's two-tailed $t$-test on GraphPad Prism 4.0 (GraphPad, La Jolla, CA).

AMP assessment. For BD2 and SLPI assessment, whole saliva was centrifuged for $5 \mathrm{~min}$ at $550 \mathrm{~g}$, and supernatant was fractionated using Centricon spin columns (Millipore/EMD) with a $100 \mathrm{kDa}$ cutoff. Filtrate was analyzed undiluted using a BD2 ELISA kit in duplicate or triplicate as volume allowed (Phoenix Pharmaceuticals, Burlingame, CA). BD2 concentrations were normalized to total protein content of centrifuged saliva. SLPI was measured in saliva samples diluted 1:5,000 using an ELISA kit (R\&D Systems, Minneapolis, MN) in triplicate. For Hst analysis, whole saliva was centrifuged at 13,000 r.p.m. $(15,700 \mathrm{~g})$ in an Eppendorf centrifuge 5415R for $10 \mathrm{~min}$ and supernatants reconstituted in 5\% acetic acid. After centrifugation for $10 \mathrm{~min}$, protein concentrations of acidic extractable proteins were measured by bicinchoninic acid assay. Samples were dried in a Vacufuge speedvac and resuspended in $3 \mathrm{M}$ urea. Samples were adjusted for total protein content by Lowry assay, and acid-urea PAGE $(15 \% \mathrm{w} / \mathrm{v})$ was performed as described previously. ${ }^{56}$ Gels were stained in Gelcode Blue (Sigma, St Louis, MO) and subjected to imaging on a Syngene Bioimaging system (Syngene Bioimaging, Frederick, MD). Histatins were identified based on migration and size compared with a loading control of purified Hsts, and quantitated by densitometry after normalization with loading control proteins.

Three-dimensional culture of HSG cells. Human submandibular gland HSG cells ${ }^{39}$ were maintained in Dulbecco's modified Eagle's medium/ Ham's F-12 (1:1), containing 5\% fetal bovine serum (Biofluids, Rockville, MD), 100 Units $\mathrm{ml}^{-1}$ penicillin, and $100 \mathrm{mg} \mathrm{ml}^{-1}$ streptomycin (Life Technologies, Gaithersburg, MD). HSG cells $\left(1 \times 10^{6}\right.$ cells per well; passage 20-40) were plated on growth factor-reduced-Matrigel, prepared as follows: $250 \mu \mathrm{l}$ of $8 \mathrm{mg} \mathrm{ml}^{-1}$; and 2:1 growth factor-reduced-Matrigel: Dulbecco's modified Eagle's medium-Ham's F-12 (1:1) medium (Becton Dickinson Labware, Franklin Lakes, NJ) was allowed to solidify in a humidified $37^{\circ} \mathrm{C}$ incubator for $1 \mathrm{~h}$ in 6 -well plates. Differentiated threedimensional cultures of HSG cells were used for cytokine treatment after incubation for 2 days. For stimulations, IL-17A (50-500 $\left.\mathrm{ng} \mathrm{m}^{-1}\right)$ 
(PeproTech, Rocky Hill, NJ) was added to HSG cultures and incubated for $48 \mathrm{~h}$ at $37^{\circ} \mathrm{C}$. Cultures were harvested for detection of Hst 5 in acidurea PAGE $(15 \% \mathrm{w} / \mathrm{v})$ by densitometry after normalization with loading controls as described for AMPs. Cytokines were purchased from PeproTech or R\&D Systems.

SUPPLEMENTARY MATERIAL is linked to the online version of the paper at http://www.nature.com/mi

\section{ACKNOWLEDGMENTS}

SLG and ME were supported by DE018822, and SLG was also supported by AR054389 and Al89768. OB was supported by DE019721. HRC was supported by a training grant to the Department of Oral Biology at SUNY Buffalo (DE007034). SMH and AFF were supported by the Intramural Research Program of NIAID, NIH, Bethesda, MD 20892. The views expressed in this article are those of the authors and do not reflect the official policy of the US. Government. We thank S. Khader, AC Peterson, $\mathrm{N}$ Hernández-Santos, and A. Ray for critical reading. We also thank S. Velichko and R. Wu for sharing insights regarding STAT3 sites within the BD2 promoter.

\section{DISCLOSURE}

SLG has received a research grant from Amgen, and consults for Kinex BioPharma and Lycera Corporation.

○ 2011 Society for Mucosal Immunology

\section{REFERENCES}

1. Dongari-Bagtoglou, A. \& Fidel, P. The host cytokine responses and protective immunity in oropharyngeal candidiasis. J. Dent. Res. 84, 966-977 (2005).

2. Scully, C., el-Kabir, M. \& Samaranayake, L.P. Candida and oral candidosis: a review. Crit. Rev. Oral. Biol. Med. 5, 125-157 (1994).

3. Fidel, P.L. \& Sobel, J.D. Protective immunity in experimental Candida vaginitis. Res. Immunol. 149, 361-373 (1998).

4. Korn, T., Bettelli, E., Oukka, M. \& Kuchroo, V.K. IL-17 and Th17 cells. Ann. Rev. Immunol. 27, 485-518 (2009).

5. Conti, H. et al. Th17 cells and IL-17 receptor signaling are essential for mucosal host defense against oral candidiasis. J. Exp. Med. 206, 299-311 (2009).

6. Hise, A.G. et al. An essential role for the NLRP3 inflammasome in host defense against the human fungal pathogen Candida albicans. Cell Host. Microbe 5, 487-497 (2009).

7. Netea, M.G., Brown, G.D., Kullberg, B.J. \& Gow, N.A. An integrated model of the recognition of Candida albicans by the innate immune system. Nat. Rev. Microbiol. 6, 67-78 (2008).

8. Ferwerda, B. et al. Human dectin-1 deficiency and mucocutaneous fungal infections. N. Engl. J. Med. 361, 1760-1767 (2009).

9. Plantinga, T.S. et al. Early stop polymorphism in human DECTIN-1 is associated with increased candida colonization in hematopoietic stem cell transplant recipients. Clin. Infect. Dis. 49, 724-732 (2009).

10. Glocker, E.O. et al. A homozygous CARD9 mutation in a family with susceptibility to fungal infections. N. Engl. J. Med. 361, 1727-1735 (2009).

11. Saijo, S. et al. Dectin-2 recognition of alpha-mannans and induction of Th17 cell differentiation is essential for host defense against Candida albicans. Immunity 32, 681-691 (2010).

12. Puel, A., Picard, C., Cypowyj, S., Lilic, D., Abel, L. \& Casanova, J.L. Inborn errors of mucocutaneous immunity to Candida albicans in humans: a role for IL-17 cytokines? Curr. Opin. Immunol. 22, 467-474 (2010).

13. Acosta-Rodriguez, E.V. et al. Surface phenotype and antigenic specificity of human interleukin 17-producing T helper memory cells. Nat Immunol. 8, 639-646 (2007).

14. Puel, A. et al. Autoantibodies against IL-17A, IL-17F, and IL-22 in patients with chronic mucocutaneous candidiasis and autoimmune polyendocrine syndrome type I. J. Exp. Med. 207, 291-297 (2010).

15. Kisand, K. et al. Chronic mucocutaneous candidiasis in APECED or thymoma patients correlates with autoimmunity to Th17-associated cytokines. J. Exp. Med. 207, 299-308 (2010).
16. Browne, S.K. \& Holland, S.M. Anti-cytokine autoantibodies explain some chronic mucocutaneous candidiasis. Immunol. Cell Biol. 88, 614-615 (2010).

17. Freeman, A.F. \& Holland, S.M. The hyper-IgE syndromes. Immunol. Allergy Clin. North Am. 28, 277-291, viii (2008).

18. Minegishi, Y. et al. Dominant-negative mutations in the DNA-binding domain of STAT3 cause hyper-lgE syndrome. Nature 448, 1058-1062 (2007).

19. Freeman, A.F. et al. Causes of death in hyper-lgE syndrome. J. Allergy Clin. Immunol. 119, 1234-1240 (2007).

20. Renner, E.D. et al. Novel signal transducer and activator of transcription 3 (STAT3) mutations, reduced $\mathrm{T}(\mathrm{H}) 17$ cell numbers, and variably defective STAT3 phosphorylation in hyper-lgE syndrome. J. Allergy Clin. Immunol. 122, 181-187 (2008).

21. Minegishi, Y. \& Karasuyama, H. Defects in Jak-STAT-mediated cytokine signals cause hyper-lgE syndrome: lessons from a primary immunodeficiency. Int. Immunol. 21, 105-112 (2009).

22. Egwuagu, C.E. STAT3 in CD4+ T helper cell differentiation and inflammatory diseases. Cytokine 47, 149-156 (2009).

23. Lin, L. et al. Th1-Th17 cells mediate protective adaptive immunity against Staphylococcus aureus and Candida albicans infection in mice. PLOS Pathog. 5, e1000703 (2009).

24. Khader, S.A., Gaffen, S.L. \& Kolls, J.K. Th17 cells at the crossroads of innate and adaptive immunity against infectious diseases at the mucosa. Mucosal Immunol. 2, 403-411 (2009).

25. Klatt, N.R. \& Brenchley, J.M. Th17 cell dynamics in HIV infection. Curr. Opin. HIV AIDS 5, 135-140 (2010).

26. Tangye, S.G., Cook, M.C. \& Fulcher, D.A. Insights into the role of STAT3 in human lymphocyte differentiation as revealed by the hyper-IgE syndrome. J. Immunol. 182, 21-28 (2009).

27. Gorr, S.U. Antimicrobial peptides of the oral cavity. Periodontol. 2009 51, 152-180 (2000).

28. Edgerton, M., Koshlukova, S.E., Lo, T.E., Chrzan, B.G., Straubinger, R.M. \& Raj, P.A. Candidacidal activity of salivary histatins. J. Biol. Chem. 273, 20438-20447 (1998)

29. Ho, A. et al. IL-17RC is required for immune signaling via an extended SEFIR domain in the cytoplasmic tail. J. Immunol. 185, 1063-1070 (2010).

30. Hibino, K., Samaranayake, L.P., Hagg, U., Wong, R.W. \& Lee, W. The role of salivary factors in persistent oral carriage of Candida in humans. Arch. Oral. Biol. 54, 678-683 (2009).

31. Ganz, T. Defensins: antimicrobial peptides of innate immunity. Nat. Rev. Immunol. 3, 710-720 (2003).

32. Aujla, S.J., Dubin, P.J. \& Kolls, J.K. Th17 cells and mucosal host defense. Semin. Immunol. 19, 377-382 (2007).

33. Kolls, J.K., McCray, P.B. Jr \& Chan, Y.R. Cytokine-mediated regulation of antimicrobial proteins. Nat. Rev. Immunol. 8, 829-835 (2008).

34. Chattopadhyay, A. et al. Salivary secretory leukocyte protease inhibitor and oral candidiasis in human immunodeficiency virus type 1-infected persons. Infect. Immun. 72, 1956-1963 (2004).

35. Williams, S.E., Brown, T.I., Roghanian, A. \& Sallenave, J.M. SLPI and elafin: one glove, many fingers. Clin. Sci. (Lond). 110, 21-35 (2006).

36. Edgerton, M., Koshlukova, S.E., Araujo, M.W., Patel, R.C., Dong, J. \& Bruenn, J.A. Salivary histatin 5 and human neutrophil defensin 1 kill Candida albicans via shared pathways. Antimicrob. Agents Chemother. 44, 3310-3316 (2000).

37. Campese, M., Sun, X., Bosch, J.A., Oppenheim, F.G. \& Helmerhorst, E.J. Concentration and fate of histatins and acidic proline-rich proteins in the oral environment. Arch. Oral. Biol. 54, 345-353 (2009).

38. Desai, K., Huston, D. \& Harriman, G. Previously undiagnosed hyper-IgE syndrome in an adult with multiple systemic fungal infections. J. Allergy Clin. Immunol. 98, 1123-1124 (1996).

39. Shirasuna, K., Sato, M. \& Miyazaki, T. A neoplastic epithelial duct cell line established from an irradiated human salivary gland. Cancer 48, 745-752 (1981).

40. Hoffman, M.P., Kibbey, M.C., Letterio, J.J. \& Kleinman, H.K. Role of laminin-1 and TGF-beta 3 in acinar differentiation of a human submandibular gland cell line (HSG). J. Cell. Sci. 109 (Part 8), 2013-2021 (1996).

41. Zheng, C., Hoffman, M.P., McMillan, T., Kleinman, H.K. \& O'Connell, B.C. Growth factor regulation of the amylase promoter in a differentiating salivary acinar cell line. J. Cell. Physiol. 177, 628-635 (1998). 
42. Miossec, P. Interleukin-17 in rheumatoid arthritis: if T cells were to contribute to inflammation and destruction through synergy. Arth. Rheum. 48, 594-601 (2003).

43. Onishi, R. \& Gaffen, S.L. IL-17 and its target genes: mechanisms of IL-17 function in disease. Immunology 129, 311-321 (2010).

44. Liang, S.C. et al. Interleukin (IL)-22 and IL-17 are coexpressed by Th17 cells and cooperatively enhance expression of antimicrobial peptides. J. Exp. Med. 203, 2271-2279 (2006).

45. Minegishi, Y. et al. Molecular explanation for the contradiction between systemic Th17 defect and localized bacterial infection in hyper-lgE syndrome. J. Exp. Med. 206, 1291-1301 (2009)

46. Kao, C.Y. et al. IL-17 markedly up-regulates beta-defensin-2 expression in human airway epithelium via JAK and NF-kappaB signaling pathways. J. Immunol. 173, 3482-3491 (2004).

47. Qian, Y. et al. Deficiency of Act1, a critical modulator of B cell function, leads to development of Sjogren's syndrome. Eur. J. Immunol. 38, 2219-2228 (2008).

48. Gross, O. et al. Syk kinase signalling couples to the Nirp3 inflammasome for anti-fungal host defence. Nature 459, 433-436 (2009).

49. Leibundgut-Landmann, S. et al. Syk- and CARD9-dependent coupling of innate immunity to the induction of $T$ helper cells that produce interleukin 17. Nat Immunol. 8, 630-638 (2007).
50. Robinson, M.J. et al. Dectin-2 is a Syk-coupled pattern recognition receptor crucial for Th17 responses to fungal infection. J. Exp. Med. 206, 2037-2051 (2009).

51. Huang, W., Na, L., Fidel, P.L. \& Schwarzenberger, P. Requirement of interleukin-17A for systemic anti-Candida albicans host defense in mice. J. Infect. Dis. 190, 624-631 (2004).

52. Duerr, R.H. et al. A genome-wide association study identifies IL23R as an inflammatory bowel disease gene. Science 314, 1461-1463 (2006).

53. Jurevic, R.J., Bai, M., Chadwick, R.B., White, T.C. \& Dale, B.A. Singlenucleotide polymorphisms (SNPS) in human beta-defensin 1: highthroughput SNP assays and association with Candida carriage in type I diabetics and nondiabetic controls. J. Clin. Microbiol. 41, 90-96 (2003).

54. Browne, S.K. \& Holland, S.M. Immunodeficiency secondary to anticytokine autoantibodies. Curr. Opin. Allergy Clin. Immunol. 10, 534541 (2010).

55. Zenewicz, L.A., Abraham, C., Flavell, R.A. \& Cho, J.H. Unraveling the genetics of autoimmunity. Cell 140, 791-797 (2010).

56. Panyutich, A. \& Ganz, T. Activated alpha 2-macroglobulin is a principal defensin-binding protein. Am. J. Respir. Cell. Mol. Biol. 5, 101-106 (1991). 